\title{
Utilization of prostate brachytherapy for low risk prostate cancer: Is the decline overstated?
}

\author{
Joseph Safdieh, MD',2, Andrew Wong, MD',2, Joseph P. Weiner, MD',2, David Schwartz, MD',2, David Schreiber, MD',2 \\ 'Department of Veteran Affairs, NY Harbor Campus, Brooklyn, NY, ${ }^{2}$ SUNY Downstate Medical Center, Brooklyn, NY, USA
}

\begin{abstract}
Purpose: Several prior studies have suggested that brachytherapy utilization has markedly decreased, coinciding with the recent increased utilization of intensity modulated radiation therapy, as well as an increase in urologist-owned centers. We sought to investigate the brachytherapy utilization in a large, hospital-based registry.

Material and methods: Men with prostate cancer diagnosed between 2004-2012 and treated with either external beam radiation and/or prostate brachytherapy were abstracted from the National Cancer Database. In order to be included, men had to be clinically staged as T1c-T2aNx-0Mx-0, Gleason 6, PSA $\leq 10.0 \mathrm{ng} / \mathrm{ml}$. Descriptive statistics were used to analyze brachytherapy utilization over time and were compared via $\chi^{2}$. Multivariate logistic regression was used to assess for covariables associated with increased brachytherapy usage.

Results: There were 89,413 men included in this study, of which 37,054 (41.6\%) received only external beam radiation, and 52,089 (58.4\%) received prostate brachytherapy. The use of brachytherapy declined over time from $62.9 \%$ in 2004 to $51.3 \%$ in 2012 ( $p<0.001)$. This decline was noted in both academic facilities $(60.8 \%$ in 2004 to $47.0 \%$ in 2012 , $p<0.001)$ as well as in non-academic facilities (63.7\% in 2004 to $53.0 \%$ in $2012, p<0.001)$. The decline was more pronounced in patients who lived closer to treatment facilities than those who lived further. The use of intensity modulated radiation therapy increased during this same time period from $18.4 \%$ in 2004 to $38.2 \%$ in $2012(p<0.001)$. On multivariate analysis, treatment at an academic center, increasing age, decreasing distance from the treatment center, and years of diagnosis from 2006-2012 were significantly associated with reduced brachytherapy usage.

Conclusions: In this hospital-based registry, prostate brachytherapy usage has declined for low risk prostate cancer as intensity modulated radiation therapy usage has increased. However, it still remains the treatment of choice for
\end{abstract} $51.3 \%$ of patients as of 2012 .

Key words: brachytherapy, low risk, NCDB, prostate cancer.

\section{Purpose}

Initially introduced in the mid-1960's, with the adoption of radioactive ${ }^{125} \mathrm{I}$ into clinical practice, prostate brachytherapy remains a highly effective treatment modality for organ confined prostate cancer [1]. With further development of the transperineal approach in the 1980's, prostate brachytherapy was adopted as a standard option for prostate cancer treatment [2]. Tumor registry studies initially revealed an increase utilization of prostate brachytherapy as a primary treatment modality in the late 1990 's and early 2000 's, as targeted radiotherapy approaches were limited during that time period [3].

However, this trend has reported to have recently reversed as newer technologies have gained acceptance, such as intensity modulated radiotherapy (IMRT), proton therapy, as well as improvement of surgical options, such as robotic assisted prostatectomy [4,5]. Others have suggested that outside issues, such as financial reimbursement, lack of physician skill proficiency in brachytherapy, as well as stage migration towards more intermediate and high grade disease, which may be less likely to include prostate brachytherapy, have played a role in this trend [6]. These issues have led to several provocative studies suggesting that prostate brachytherapy is on a steep decline.

Most of these studies reporting on the decline of prostate brachytherapy have analyzed its usage in comparison to all available treatment options. In this study we sought to utilize the National Cancer Database (NCDB) in order to evaluate the temporal trends of prostate brachytherapy in the setting of men with low risk disease who have elected to undergo treatment with radiotherapy. By limiting our cohort to those who are most likely to select brachytherapy (patients with low risk disease) and have selected radiation therapy over observation or surgery, we sought to obtain a more nuanced view into whether prostate brachytherapy is really on the decline compared to external beam radiation. 


\section{Material and methods}

The NCDB is a hospital-based registry that is the joint project of the American Cancer Society and the Commission on Cancer of the American College of Surgeons. It is estimated that $70 \%$ of all diagnosed malignancies in the United States are captured by facilities participating in this registry and reported to the NCDB. The Commission on Cancer's NCDB and the hospitals participating in the NCDB are the source of the de-identified data used in this study. However, they have not verified and are not responsible for the statistical validity or conclusions derived by the authors of this study. Exemption was obtained from the New York Harbor Veterans Affairs Committee for Research and Development prior to the initiation of this study.

Men with prostate cancer diagnosed between 20042012 and treated with either external beam radiation and/ or prostate brachytherapy were abstracted from the NCDB. In order to be included, men had to be clinically staged as $\mathrm{T} 1 \mathrm{c}-\mathrm{T} 2 \mathrm{aNx}-0 \mathrm{Mx}-0$ adenocarcinoma of the prostate, Gleason score $\leq 6$, and prostate specific antigen $\leq 10.0 \mathrm{ng} / \mathrm{ml}$, who were treated with radiation therapy were included in this analysis. This limited inclusion to men with low risk prostate cancer as classified by the National Comprehensive Cancer Network. In order to accurately assess treatment selection by facility type, we further limited analysis to those who received all of their therapy in one center, which is suggested by the NCDB for analyses that include facility type as one of the covariables.

Descriptive statistics were used to analyze brachytherapy utilization over time, as well as by other factors such as facility type, insurance status, and race. Comparisons were made via $\chi^{2}$, Fisher's Exact test, and Mann Whitney test where appropriate. Multivariate logistic regression was also used to assess for predictors of brachytherapy usage. The variables analyzed in the multivariate model included age grouping ( $<60$ years, $60-70$ years, $>70$ years), year of diagnosis (2004 through 2012 in single year increments), race (white, black, other), distance from treatment center (divided into 4 quartiles), facility type (academic, non-academic), and insurance type (none, private insurance, Medicaid, Medicare, other government, unknown). Significant values were defined as those with a $p$-value $<0.05$. Statistical analysis was performed using SPSS, Version 23 (IBM Inc, Armonk, NY, USA).

\section{Results}

There were 89,143 men included in this study. Of these, $37,054(41.6 \%)$ received external beam radiation alone, and 52,089 received brachytherapy (58.4\%), which consisted of 50,248 men who received brachytherapy alone, and 1,841 men who received brachytherapy in addition to external beam radiation. The median age was 66 years (interquartile range 61-72 years). For those who received external beam radiation, the median dose was 75.6 Gy (interquartile range 74-78 Gy). The radiation dose for those who received brachytherapy was not recorded. Men who received brachytherapy tended to be younger, live further away from the treatment center, have private insurance, and were white. Further details regarding external beam radiation and brachytherapy usage, as well as a comparison between the groups are available in Table 1.

Brachytherapy usage declined every year from 20042012 , with the exception of 2010, when there was a small relative increase compared to 2009. Nevertheless, brachytherapy remained the more commonly utilized modality overall. In 2004, brachytherapy was utilized $62.9 \%$ of the time whereas by 2012 this was reduced to $51.3 \%$ of the time. Figure 1 depicts the brachytherapy utilization by year of diagnosis.

The overall utilization of brachytherapy differed significantly by the distance from the treatment site. As noted in Table 1, those in the closest quartile were treated with brachytherapy $50.2 \%$ of the time, whereas those who were furthest away received brachytherapy 69.2\% of the time. For those men who were furthest away from the treatment center, the relative decline of brachytherapy was less steep, from $71.1 \%$ to $65.3 \%$. In contrast, for those who were closest to the treatment center, brachytherapy declined from $55.5 \%$ to $40.3 \%$.

The decline in brachytherapy was also a bit steeper for men treated at an academic center compared to a non-academic center. In 2004, brachytherapy was utilized $60.8 \%$ of the time at academic centers and this declined to $47.0 \%$ by 2012 . In non-academic centers, brachytherapy was utilized $63.7 \%$ of the time in 2004 and this declined to $53.0 \%$ by 2012. This was despite the fact that the relative percentage of men treated at an academic center increased from $26 \%$ to $31 \%$ with increasing distance from the treatment center. Additionally, for men treated in the furthest quartile, brachytherapy declined from $70.8 \%$ in 2004 to $62.4 \%$ in 2012 at academic centers, whereas during this same time period the decline change in utilization was $71.3 \%$ in 2004 to $66.5 \%$ in 2012 at non-academic centers.

On multivariate analysis, increasing distance from the treatment center was associated with an increased likelihood of brachytherapy utilization, as well as the presence of private insurance, Medicare, or government insurance. In contrast, older age, race other than white, year of diagnosis between 2006-2012, and treatment at an academic program was associated with a lower likelihood of receiving brachytherapy. Further details are available in Table 2.

\section{Discussion with Conclusions}

Initial population-based studies demonstrated an increase in utilization of prostate brachytherapy. A study published from the Surveillance, Epidemiology and End Results (SEER) database, demonstrated an increase in utilization of brachytherapy between 1986 and 2004 from $0 \%$ to $29 \%$. This increase was more pronounced in the well and moderately differentiated cancers than in the lower grade cancers [3]. Hamilton et al. identified more than 2000 patients diagnosed with prostate cancer between 1998-2002. When they included all treatment options, there was an increase in the use of brachytherapy from $15 \%$ to $17.7 \%$. This change was more pronounced in the $60-64$ age group $(19.6 \%$ to $25.2 \%)$ [7].

Our study used the NCDB database to identify almost 90,000 men diagnosed with low risk prostate cancer between 2004-2012 who were treated with radiation 
Table 1. Patient characteristics of those with low risk prostate cancer receiving external beam radiation therapy or brachytherapy

\begin{tabular}{|c|c|c|c|}
\hline Factor & EBRT $(n=37,054)$ & Brachytherapy $(n=52,089)$ & $P$-value \\
\hline Age (median) & 68 years & 65 years & $<0.001$ \\
\hline Distance from treatment center & & & $<0.001$ \\
\hline First quartile (closest) & $11,144(49.8 \%)$ & $11240(50.2 \%)$ & \\
\hline Second quartile & $9,804(45.3 \%)$ & $11,829(54.7 \%)$ & \\
\hline Third quartile & 8,993 (41.2\%) & $12,859(58.8 \%)$ & \\
\hline Last quartile (furthest) & $6,666(30.4 \%)$ & $15,228(69.6 \%)$ & \\
\hline Race & & & $<0.001$ \\
\hline White & $29,617(40.5 \%)$ & $43,593(59.5 \%)$ & \\
\hline Black & $5,893(47.0 \%)$ & $6,640(53.0 \%)$ & \\
\hline Other & $1,544(45.4 \%)$ & $1,856(54.6 \%)$ & \\
\hline Facility type & & & $<0.001$ \\
\hline Non-academic program & $26,144(40.9 \%)$ & $37,821(59.1 \%)$ & \\
\hline Academic Program & $10,910(43.3 \%)$ & $14,268(56.7 \%)$ & \\
\hline Insurance & & & $<0.001$ \\
\hline None & $494(54.3 \%)$ & $416(45.7 \%)$ & \\
\hline Private insurance & $13,664(35.7 \%)$ & $24,620(64.3 \%)$ & \\
\hline Medicaid & $924(56.7 \%)$ & $706(43.3 \%)$ & \\
\hline Medicare & $20,550(45.3 \%)$ & $24,842(54.7 \%)$ & \\
\hline Other Government & $722(44.9 \%)$ & $885(55.1 \%)$ & \\
\hline Unknown & 700 (53.0\%) & 620 (47.0\%) & \\
\hline
\end{tabular}

EBRT-external beam radiation therapy

treatment. In total, brachytherapy utilization outnumbered external beam ( $58.4 \%$ vs. $41.6 \%)$. However, when trended by year of diagnosis, brachytherapy utilization fell 12\% between 2004 and 2012. On multivariate analysis, increased brachytherapy utilization was associated with increased distance from the treatment center, private insurance, Medicare or government insurance; while older ages, race other than white, years of diagnosis between 2006-2012 and treatment at an academic program was associated with increased use of external beam radiation therapy (EBRT).

These findings of decreasing prostate brachytherapy use are corroborated by other database studies. A recently reported SEER analysis observed treatment practices of patients with any low, intermediate, or high risk disease diagnosed between 2004 and 2009, and were treated with radiation therapy. In that study, EBRT monotherapy increased from $55.8 \%$ to $62 \%$, while all brachytherapy usage decreased from $44.2 \%$ to $38.0 \%$. This decrease was significantly more pronounced in low risk patients when compared to other risk groups $(p<0.0001)$ [5]. Interestingly, they found a decrease of brachytherapy utilization in white patients, whereas our study demonstrated that

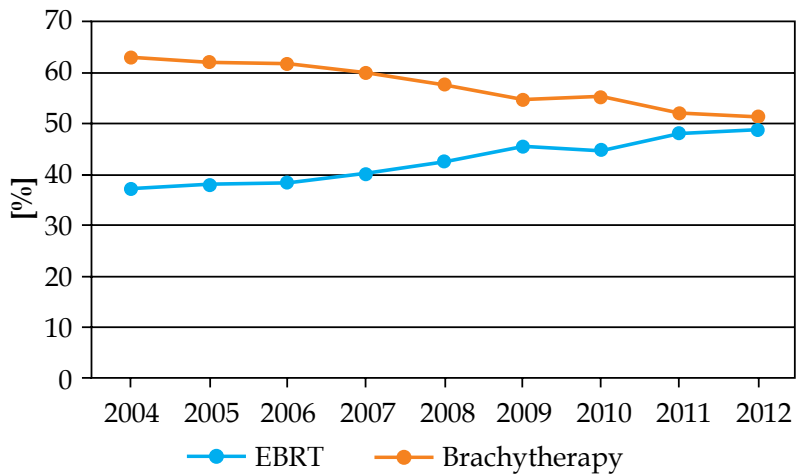

Fig. 1. Brachytherapy utilization 2004-2012

the odds ratio for brachytherapy was $0.80(p<0.001)$ for black patients and $0.86(p<0.001)$ for other ethnicities.

An additional recently published study used the NCDB and observed overall treatment trends in patients diagnosed between 1998 and 2010. During that time period they identified an increase in the use of surgery from $46.1 \%$ in 1998 to $59.1 \%$ in 2010 , while they noted a decline in all other treatment modalities. They found that brachytherapy use peaked in 2002 at $16.7 \%$ and then de- 
Table 2. Multivariate logistic regression analysis for brachytherapy usage

\begin{tabular}{|c|c|c|}
\hline Factor & Odds ratio $(95 \% \mathrm{Cl})$ & $P$-value \\
\hline \multicolumn{3}{|l|}{ Age grouping } \\
\hline$<60$ years & 1 & \\
\hline $60-70$ years & $0.73(0.70-0.76)$ & $<0.001$ \\
\hline$>70$ years & $0.48(0.46-0.50)$ & $<0.001$ \\
\hline \multicolumn{3}{|c|}{ Year of diagnosis } \\
\hline 2004 & 1 & \\
\hline 2005 & $0.96(0.91-1.01)$ & 0.13 \\
\hline 2006 & $0.93(0.88-0.99)$ & 0.01 \\
\hline 2007 & $0.87(0.83-0.92)$ & $<0.001$ \\
\hline 2008 & $0.77(0.73-0.82)$ & $<0.001$ \\
\hline 2009 & $0.67(0.63-0.71)$ & $<0.001$ \\
\hline 2010 & $0.68(0.64-0.72)$ & $<0.001$ \\
\hline 2011 & $0.60(0.56-0.63)$ & $<0.001$ \\
\hline 2012 & $0.57(0.53-0.61)$ & $<0.001$ \\
\hline \multicolumn{3}{|l|}{ Race } \\
\hline White & 1 & \\
\hline Black & $0.80(0.77-0.83)$ & $<0.001$ \\
\hline Other & $0.86(0.80-0.93)$ & $<0.001$ \\
\hline
\end{tabular}

Distance from treatment center

\begin{tabular}{lcc}
\hline First quartile (closest) & 1 & \\
\hline Second quartile & $1.18(1.13-1.22)$ & $<0.001$ \\
\hline Third quartile & $1.36(1.31-1.41)$ & $<0.001$ \\
\hline Last quartile (furthest) & $2.22(2.13-2.31)$ & $<0.001$ \\
\hline
\end{tabular}

\begin{tabular}{|c|c|c|}
\hline \multicolumn{3}{|l|}{ Facility type } \\
\hline Non-academic program & 1 & \\
\hline Academic program & $0.87(0.84-0.90)$ & $<0.001$ \\
\hline \multicolumn{3}{|l|}{ Insurance } \\
\hline None & 1 & \\
\hline Private insurance & $2.07(1.80-2.37)$ & $<0.001$ \\
\hline Medicaid & $1.01(0.85-1.19)$ & 0.92 \\
\hline Medicare & $1.72(1.50-1.98)$ & $<0.001$ \\
\hline Other Government & $1.39(1.17-1.65)$ & $<0.001$ \\
\hline Unknown & $1.12(0.94-1.34)$ & 0.19 \\
\hline
\end{tabular}

clined to a low of $8 \%$ in 2010 . Low risk patients exhibited the sharpest decline in brachytherapy use by $9.4 \%$ [4]. In our study, we noted that prostate brachytherapy still remains an important treatment of choice for many patients with $>50 \%$ of men undergoing radiation therapy choos- ing brachytherapy in 2012. However, given the current trend, one would expect this to continue to decline into the next decade.

The utilization rates in our study are higher than in other reports, which is likely due to different methodologies. The SEER database study looked at global declining rates of brachytherapy amongst all risk groups, but did not detail the relative usage of brachytherapy compared to EBRT by risk group [5]. The aforementioned NCDB study compared brachytherapy usage to all other modalities, including surgery, hormones, and no treatment. They identified that all modalities appeared to be decreasing in utilization as surgery increased. In addition, they excluded several brachytherapy variables due to questions regarding the fidelity of those variables at the time of their analysis, which may have led to some degree of underestimation of brachytherapy usage [4]. In fact, a prior SEER based study using more similar methodology to ours reported that prostate brachytherapy utilization was $56.1 \%$ for low risk patients, which is consistent with our findings [8].

Regardless of the precise comparative methodologies used, the consistent findings from all of these studies are that prostate brachytherapy appears to be declining. This is somewhat surprising, as many studies to date demonstrate an increase in prostate-specific antigen (PSA) progression free survival with brachytherapy versus other modalities. The Prostate Cancer Results Study Group recently published a study, which included almost 850 different publications and found that compared to EBRT or radical prostatectomy, brachytherapy was associated with the most sustained PSA progression free survival over 12 years [9]. Another retrospective analysis looked at almost 8,000 low and intermediate risk patients treated in Canada with radiotherapy, and found that the use of brachytherapy led to statistically significant improvement in PSA progression free survival, with a hazard ratio of 4.58 for intermediate risk patients and 2.90 for low risk patients [10]. The recently reported ASCENDE-RT trial showed an increased survival with the addition of brachytherapy to EBRT in intermediate and high risk patients [11].

Several possibilities have been suggested to explain this trend, including increased utilization of robotic assisted surgery [12], fewer practitioners being trained, and possibly financial incentives [5]. With IMRT paying almost $\$ 15,000$ per patient, private physicians may be incentivized to offer longer treatment and avoid pushing brachytherapy [13]. The increase in urology owned centers further push the treatment preference towards IMRT [14]. Prostate cancer is among the top five most expensive cancers in the United States, with approximately $\$ 12$ billion spent annually [15]. It is estimated that approximately 70,110 low risk prostate cancers will be diagnosed in the United States within the next ten years, and the cost to treat them with low-dose-rate (LDR) brachytherapy would be $\$ 18.3$ billion, $\$ 32.2$ billion for high-dose-rate (HDR) brachytherapy and $\$ 54$ billion for IMRT [16]. In the current era of increased emphasis on cost containment, these financial issues will likely become an urgent healthcare issue in the United States. 
Although financial incentive seems like a strong driver for the decrease in brachytherapy utilization, our study demonstrated a much larger decline of brachytherapy utilization in academic centers, where one would expect financial incentive to play less of a role. This finding suggests that there may be other factors driving these changes and is of particular concern as academic centers provide most of resident training throughout the county. Recent Association of Residents in Radiation Oncology (ARRO) survey reported an enormous decrease in resident reported clinical experience in brachytherapy from $88 \%$ in the $2005-2008$ survey to $15-30 \%$ in the $2013-2015$ survey [17]. Another recently published study determined that the number of academic centers performing $\leq 1$ brachytherapy procedure a month has increased from $56.4 \%$ to $73.7 \%$ [18]. This translates into practicing physicians who are uncomfortable with performing the procedure, limiting treatment options offered to patients, and will likely lead to further decline through the years. It is incumbent on academic centers to take an increased responsibility for this issue, in order to prevent a nationwide drought of brachytherapy efficient physicians.

We also noted that distance clearly plays a role in brachytherapy utilization, as there was an increase in utilization in patients who lived further from treatment facilities. This was most pronounced in those that lived furthest from treatment facilities, where the brachytherapy peaked to $70 \%$ in those patients. The steepest decline in brachytherapy remains patients that live closest to treatment facilities. As typical EBRT treatment require 8-9 weeks of therapy, it would be taxing for one that lives far away from the treatment facilities to undergo daily treatment for that time period, further favoring brachytherapy in that group.

As with all tumor registry studies, limitations to this study include selection bias, incomplete data, and coding errors. The NCDB in particular is a hospital based database, and therefore if patients either received external beam at an outpatient facility and received brachytherapy at an NCDB hospital or vice versa, it may lead to an over or underestimation of the findings. For example, if a patient received external beam at a hospital but brachytherapy at a private institution, this may have underestimated true brachytherapy utilization. In attempt to account for that potential bias, only patients who received all of their treatment at the same facility were included in this analysis.

In conclusion, prostate brachytherapy remains for now the most commonly selected radiation treatment for low risk disease. However, there has been a consistent trend of reduced brachytherapy usage over the last decade, which if it continues may eventually lead to marginalization of prostate brachytherapy over time. Given its favorable clinical and cost effectiveness, further studies should be directed to determine barriers to brachytherapy, particularly at academic centers, in order to reduce this trend.

\section{Disclosure}

Authors report no conflict of interest.

\section{References}

1. Beyer DC. The evolving role of prostate brachytherapy. Cancer Control 2001; 8: 163-170.
2. Holm HH, Juul N, Pedersen JF et al. Transperineal ${ }^{125}$ iodine seed implantation in prostatic cancer guided by transrectal ultrasonography. J Urol 1983; 130: 283-286.

3. Jani AB, Johnstone PA, Liauw SL et al. Prostate cancer modality time trend analyses from 1973 to 2004: A Surveillance, Epidemiology, and End Results registry analysis. Am J Clin Oncol 2010; 33: 168-172.

4. Martin JM, Handorf EA, Kutikov A et al. The rise and fall of prostate brachytherapy: use of brachytherapy for the treatment of localized prostate cancer in the National Cancer Data Base. Cancer 2014; 120: 2114-2121.

5. Mahmood U, Pugh T, Frank S et al. Declining use of brachytherapy for the treatment of prostate cancer. Brachytherapy 2014; 13: 157-162.

6. Greenberg DC, Wright KA, Lophathanon A et al. Changing presentation of prostate cancer in a UK population - 10 year trends in prostate cancer risk profiles in the East of England. Br J Cancer 2013; 109: 2115-2120.

7. Hamilton AS, Albertsen PC, Johnson TK et al. Trends in the treatment of localized prostate cancer using supplemented cancer registry data. BJU Int 2011; 107: 576-584.

8. Schreiber D, Chen SC, Rineer J et al. Racial and socioeconomic disparities in the selection of prostate brachytherapy. J Contemp Brachytherapy 2013; 5: 139-143.

9. Grimm P, Billiet I, Bostwick D et al. Comparative analysis of prostate-specific antigen free survival outcomes for patients with low, intermediate and high risk prostate cancer treatment by radical therapy. Results from the Prostate Cancer Results Study Group. BJU Int 2012; 109 Suppl 1: 22-29.

10. Smith GD, Pickles T, Crook J et al. Brachytherapy improves biochemical failure-free survival in low- and intermediate-risk prostate cancer compared with conventionally fractionated external beam radiation therapy: a propensity score matched analysis. Int J Radiat Oncol Biol Phys 2015; 91: 505516.

11. Morris WJ, Tyldesley S, Pai HH et al. ASCENDE-RT*: A multicenter, randomized trial of dose-escalated external beam radiation therapy (EBRT-B) versus low-dose-rate brachytherapy (LDR-B) for men with unfavorable-risk localized prostate cancer. 2015 ASCO Annual Meeting. http:/ / meetinglibrary. asco.org/content/141698-159. Accessed September 10, 2015.

12. Stitzenberg KB, Wong $Y N$, Nielsen ME et al. Trends in radical prostatectomy: centralization, robotics, and access to urologic cancer care. Cancer 2012; 118: 54-62.

13. Nguyen PL, Gu X, Lipsitz SR et al. Cost implications of the rapid adoption of newer technologies for treating prostate cancer. J Clin Oncol 2011; 29: 1517-1524.

14. Mitchell JM. Urologists' use of intensity-modulated radiation therapy for prostate cancer. N Engl J Med 2013; 369: 1629-1637.

15. Mariotto AB, Yabroff KR, Shao Y et al. Projections of the cost of cancer care in the United States: 2010-2020. J Natl Cancer Inst 2011; 103: 117-128.

16. Durkee BY, Buyyounouski MK. The case for prostate brachytherapy in the Affordable Care Act era. Int J Radiat Oncol Biol Phys 2015; 91: 465-467.

17. Nabavizadeh N, Burt LM, Mancini BR et al. Results of the 2013-2015 Association of Residents in Radiation Oncology Survey of Chief Residents in the United States. Int J Radiat Oncol Biol Phys 2016; 94: 228-234.

18. Orio PF, Nguyen PL, Buzurovic I et al. Prostate brachytherapy case volumes by academic and non-academic practices: Implications for future residency training. Int J Radiat Oncol Biol Phys 2016 Jul 21. [Epub ahead of print]. 